\title{
Identifying India's Dual Nutrition Burden: Role of Body Mass Index Quick Screening Tool
}

\author{
SANGita YADAV \\ Department of Pediatrics, Maulana Azad Medical College and L N Hospital, University of Delhi, New Delhi 110002, India. \\ drsangeetayadav18@gmail.com
}

A dual nutrition burden of undernutrition with rise in childhood obesity was recognized in India in the latter decade of the twentieth century [1].Yes, the pendulum has swung from the era of undernutrition from 1960s-80s to the era of plenty, leading to over-nutrition from late 90 s till the present. The healthcare systems are now focusing on the burden of obesity in childhood because of its long term consequences of non-communicable diseases in adulthood [2]. However, surveillance for undernutrition is imperative as part of the life cycle approach to ensure optimum health at birth and later in life. The Prime Minister's Overarching Scheme for Holistic Nourishment (POSHAN) Abhiyaan, a multi-ministerial convergence mission, was initiated in 2018 by the Government of India to ensure adequate nutrition of pregnant women and lactating mothers and holistic development of children, with a vision to attain malnutrition-free India by 2022 [3] .

Body mass index (BMI) is currently the best simple available anthropometric estimate of fatness for public health purposes, proposed first by Cole, et al. [4] in children in 1979, which adjusts weight for both height and age. The validity of anthropometric data as a proxy for body fat identifies children at risk and correlates better with measures of body fat mass [4]. The International Obesity Taskforce (IOTF) pooled data from six international BMI references to define the centile cut offs at 18 years of age to match the adult cut offs of 25 $\mathrm{kg} / \mathrm{m}^{2}$ and $30 \mathrm{~kg} / \mathrm{m}^{2}$ for overweight and obesity. However, studies conducted in India showed the IOTF reference classified participants as having a lower weight and concluded that IOTF criteria were not suitable for Indian and South Asian children [5]. Thus, lower BMI cut-offs of $23 \mathrm{~kg} / \mathrm{m}^{2}$ and $25 \mathrm{~kg} / \mathrm{m}^{2}$ have been suggested by the World Health Organization (WHO) and IOTF for Asian Indian adults for overweight and obesity, respectively but these are not applicable for children and adolescents [6]. Over the years, there has been a lack of consensus on the various cut-points or definitions used to classify obesity and overweight in children and adolescents. This makes it difficult to interpret and compare the global or national prevalence rates. For children and adolescents, overweight and obesity are usually defined using age and gender specific nomograms of BMI. The Indian Academy of Pediatrics recommends the revised growth charts for height, weight and BMI for assessment of growth of Indian children aged 5-18 year. Overweight and obesity have been defined using adult equivalent of $23 \mathrm{~kg} / \mathrm{m}^{2}$ and $27 \mathrm{~kg} / \mathrm{m}^{2}$ cut-offs presented in BMI charts in children from 5-18 years [7]. Higher prevalence of obesity and overweight was reported with IAP 2015 reference than IOTF and WHO 2007 standards in the age group of 8-18 years, with good agreement [8].

With the need to identify over nutrition early, it is important to calculate and plot BMI at least once a year in all children and adolescents, and identify weight patterns relative to linear growth. The use of charts helps track BMI to give guidance. Monitoring of BMI is; however, often overlooked in routine clinical practice unless the issue is recognized by parents, which may be rather late at times. Many parents would need an interpretation of their child's BMI and assessment of their child's health risks. Defining one or more cut-off points on the BMI chart determines the advice to be communicated to the parents at a stage when interventions might be easier.

The 'ELIZ health path for adolescents and adults (EHPA)' novel growth assessment chart was designed to plot the height on the $\mathrm{X}$ axis and weight on the $\mathrm{Y}$ axis and then read the BMI from the right margin in accordance with the International Obesity Task Force (IOTF) recommendations for the various age groups [9]. The lower and the higher cut-off indicators on this chart were found appropriate for preliminary screening of a large number of children and adolescents in the community setting $[9,10]$.

In this issue Khadilkar, et al. [11] report on the development of a graphic tool for the BMI cut offs, without need for calculating BMI, for screening from 8 years onwards for underweight, overweight and obesity, 
which complements the existing IAP 2015 charts. They validated the tool using de-identified data on children from school health surveys and found that the BMI tool had a sensitivity of $100 \%$ for both boys and girls with a specificity of $88.9 \%$ and $82.4 \%$ for boys and girls, respectively for underweight. The sensitivity and specificity was $95.7 \%$ and $85.7 \%$ for boys, and $95.7 \%$ and $89.7 \%$ for girls, respectively for detection of overweight and obesity. Thus, the tool demonstrated high sensitivity and specificity for screening children for underweight, overweight and obesity against IAP BMI charts. They also observed that the tool may wrongly categorize children at extreme ends of height for age. However, larger studies with a bigger sample size are required for validation and generalization of the tool. The tool is gender-specific and is based on height and weight, which eliminates the need for calculation of BMI, and may help pediatricians to rapidly screen for any changes in BMI in a busy clinical practice.

Efforts to decrease the existing nutritional scenario of dual burden of undernutrition alongside emerging over nutrition should be a top priority. The present narrative shows that overweight and obesity rates in children and adolescents are increasing among the higher socioeconomic groups and in the lower income groups where underweight still remains a major concern. No country can aim to attain economic and social development goals without addressing the issue of malnutrition. This suggests the need for a balanced and sensitive approach addressing economic and nutrition transitions to effectively tackle this double burden paradox in India. Since the comorbidities of undernutrition, low birth weight, and overweight/obesity with associated noncommunicable diseases co-exist in India, it is important to integrate nutritional concerns in developmental policies.

The key to long-term solutions lies in prevention with a proactive approach.BMI performs moderately well as a proxy for nutritional indicators and is the best available tool for monitoring progress in the campaign for identifying malnutrition in India. A robust quality assured anonymized data collection and analysis system can provide national and local data that would inform the planning and evaluation of intervention programs. BMI can be an effective screening test for undernutrition; however, the statistical cut-off points are inherently arbitrary and must be followed up by a more detailed evaluation to assess the risks and plan intervention.

Funding: None; Competing interest: None stated.

\section{REFERENCES}

1. Mathur P, Pillai R. Overnutrition: Current scenario and combat strategies. Indian J Med Res. 2019;149:695-705

2. India State-Level Disease Burden Initiative Malnutrition Collaborators. The burden of child and maternal malnutrition and trends in its indicators in the states of India: the Global Burden of Disease Study 1990-2017 [published correction appears in Lancet Child Adolesc Health. 2019 Sep 30]. Lancet Child Adolesc Health. 2019;3:855-70.

3. NITI Aayog. POSHAN Abhiyaan. Available at URL: https://niti.gov.in/poshan-abhiyaan. Accessed September 12, 2020.

4. Hall DMB, ColeTJ. What use is the BMI? Arch Dis Child 2006; 91:283-6.

5. Ranjani H, MehreenTS, Pradeepa R, et al. Epidemiology of childhood overweight \& obesity in India: A systematic review. Indian J Med Res. 2016;143:160-74.

6. Cole TJ, Lobstein T. Extended international (IOTF) body mass index cut-offs for thinness, overweight and obesity. Pediatr Obes. 2012; 7:284-94.

7. Indian Academy of Pediatrics Growth Charts Committee. Khadilkar V, Yadav S, Agrawal KK, et al. Revised IAP Growth Charts for Height, Weight and Body Mass Index for 5- to 18-Year-Old Indian Children. Indian Pediatr. 2015;52:47-55.

8. Chudasama RK, Eshwar T, Eshwar ST, Thakrar D. Prevalence of Obesity and Overweight Among School Children Aged 8-18 Years in Rajkot, Gujarat. Indian Pediatr. 2016;53:743-4.

9. Elizabeth KE. A novel growth assessment chart for adolescents. Indian Pediatr. 2001; 38:1061-4.

10. Elizabeth KE, Muraleedharan M. Three-in-one weight, height and body mass index charts for children and adults. J Trop Pediatr. 2003;49: 224-7.

11. Khadilkar V, Lohiya N, Chiplonkar S, Khadilkar A. Body mass index quick screening tool for IAP 2015 growth charts. Indian Pediatr. 2020;57:904-06. 